\title{
An automotive Side-View system based on Ethernet and IP
}

\author{
Alexander Camek and Christian Buckl \\ Cyber-Physical Systems \\ fortiss $\mathrm{GmbH}$ \\ Guerickestrasse 25 \\ 80805 München, Germany \\ \{camek,buckl\}@fortiss.org
}

\author{
Pedro Sebastiao Correia \\ I/AEV-22 \\ Audi Electronic Venture GmbH \\ Sachsstrasse 18 \\ 85080 Gaimersheim, Germany \\ pedro-sebastiao.correia@audi.de
}

\author{
Prof. Dr.-Ing. Alois Knoll \\ Fakultät für Informatik \\ Technische Universität München \\ Boltzmannstrasse 3 \\ 85748 Garching bei München, Germany \\ knoll@in.tum.de
}

\begin{abstract}
Emerging driver assistance systems pose new requirements on the communication protocols. This is especially true for assistance systems based on cameras as sensors. This paper discusses the challenges of such systems using the Side View system, which uses five cameras as input, as an example. Standard and potential communication protocols are evaluated with respect to these challenges in this paper. The paper argues why Ethernet and IP form a suitable basis for new driver assistance systems and describe a first prototypical implementation of the Side View system.
\end{abstract}

Keywords-CAN; LIN; TTP/C; FlexRay; LVDS; MOST; Ethernet; AVB; IP; automotive; driver assistance; Side-View

\section{INTRODUCTION}

Many automobile manufacturers equips their latest vehicles with modern driver assistance systems. At the very beginning only radar systems were used. These systems are still part of the cars because of their low costs, reliability and the small bandwidth. Modern driver assistance systems put stress on camera based systems similar to human eyes.

An example for such a system is a Side-View which uses cameras to generate a panoramic view. With such a view a driver can easily survey the situation around a vehicle.

The main challenge when integrating this new driver assistance systems in the car is the required bandwidth. Modern camera based driver assistance applications need a high resolution to get every feature in an image. This allows the use of better image processing algorithms, e.g. for road sign recognition or pedestrian recognition. To get better results the image processing community prefers raw image data instead of compressed data. To transfer raw image data with a high resolution a reliable communication bus with a high bandwidth is needed.

The main contribution of this paper is an analysis of current automotive networks with respect to the needs of modern camera based driver assistance systems. The paper starts with a description of Side-View, its use use case as well as a description of our prototypical implementation in section II. The description is followed by an overview and evaluation of currently used automotive network systems, as

This work was funded by BMBF-Project "Sicherheit in eingebetteten IP-basierten Systemen” (SEIS) [1]. well as potential ones, in section III. The paper ends with a summary in section $\mathrm{V}$.

\section{Side-VIEW: UsE CASE}

The main use case of Side-View is to check the environment of a vehicle. In a repair station a mechanic could for example use a tablet PC (e.g. iPad) to get all diagnostic information from the vehicle. During some of these tests, it has to be guaranteed that nobody is near the vehicle. The Side-View helps to ensure this condition: the mechanics can use the tablet PC to connect over the repair station system to the internal system and to get the camera data. In this scenarios, the repair station system is a server based outside the vehicle. It will connect to the vehicle's internal Car-PC through a gateway by demand. This allows an user to get access to each camera in the car, to switch on or off each camera, to analyze and monitor traffic given on the Ethernet network or to configure all vehicle build-in switches.

Another use case is the possibility to monitor the vehicle. Based on Side-View, a user may check if everything is all right or the system can inform the user when an unauthorized person approaches it. In this scenario the user can connect to a specific server by using his smart phone, tablet or computer. The server can be provided by an OEM or by a provider. After the connection is established a preview of all cameras will be displayed. By clicking on a specific view, a camera can be chosen and the image can be enlarged. The user might want to use Side-View for different reasons: to monitor some pets or babies left in the vehicle or to analyze the situation in case of an accident. Additionally rescue teams or action forces can use such a system to get information. Rescue teams can get information about what happened, how many occupants and where occupants were seated when a severe accident happened. Action forces might be allowed to get access to the in-car camera to identify the car hijacker or to see where the hijacker is currently driving.

\section{AnAlysis OF AUTOMOTIVE COMMUNiCATION BUSES}

In this part all state-of-the art automotive communication buses and in addition prospective protocols, such as Ethernet and AVB, are described and evaluated with respect to their 


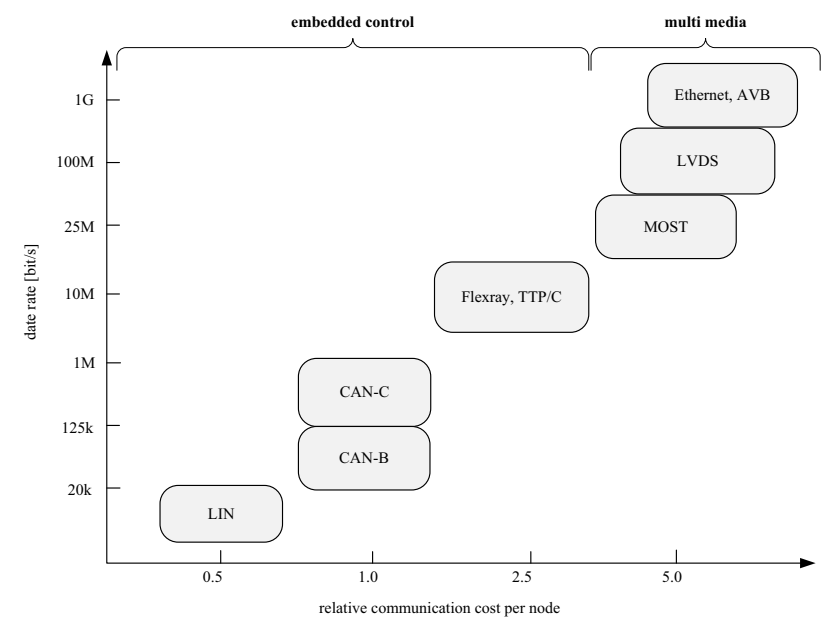

Figure 1. Bandwidth and cost relationship of automotive networks

suitability for the use case. A first classification of these technologies is given in figure 1. First a short introduction of every communication network is given with their specific generic advantages and disadvantages. In the following, we compare the protocol with the requirements of modern camera based driver assistance systems. The outcome is a statement whether the communication system supports camera based systems or not.

Analyzing communication technologies for there suitability is nothing new. However, most of the analysis were very function-specific. An example is [2], where network systems have been examined to transport power informations for power management. In the area of modern camera based driver assistance systems no such analysis exists to our knowledge.

\section{A. Controller Area Network (CAN) bus}

CAN bus [3] was first developed by Bosch Rexroth as an automotive solution, but nowadays also used in the automation domain. The specification only defines the physical layer (PHY), bit transfer encoding, the transport of telegrams and error correction. Beside that one variant is an enhancement of CAN to TTCAN (Time Triggered CAN). It supports real time properties and uses clock synchronization. Another variant is the VAN (Vehicle Area Network). The main difference are a direct reply to a telegram, a reduction to 12 bit identifier, an extension of the arbitration field into the data field and the possibility of broadcast. Both variants are used for specific use cases, but not for camera based driver assistance systems.

1) Advantages: CAN is a dual wire multi-master system with message prioritization. The system configuration is flexible and the data transfer supports error detection and correction mechanism. This allows checks whether errors are temporarily or permanent. If a permanent error is detected the CAN node will be shut down automatically. The maximum transfer speed is $1 \mathrm{Mbit} / \mathrm{s}$ by $1 \mathrm{Mbaud}$. Given a load of $50 \%$ and a protocol overhead of $50 \%$ the multi-master system has got a payload of $25 \mathrm{kbyte} / \mathrm{s}$. Most automobile manufacturer use only a maximum transfer speed of 500 $\mathrm{kbit} / \mathrm{s}$.

2) Disadvantages: A maximum transfer speed of 1 Mbit/s is only possible if the maximum cable length is not exceeded. The same constraint holds for branch lines. Additionally both ends need a terminal resistor to allow a high transfer speed. Worst case transmission time and jitter of CAN messages are not known.

3) Recommendation: The only possibility to use CAN in a camera based system is to connect each camera with an individual ECU which calculates a feature or object list for each provided image. However, such a setup would be very inefficient for modern driver assistant applications due to the required effort for cabling. As mentioned before, CAN has got a maximum payload of $25 \mathrm{kbyte} / \mathrm{s}$. That would allow to transmit an 8 bit gray scale image with a maximum resolution of $32 \times 32$ given a slow frame rate of 25 images per second. Therefore the bandwidth of CAN is too low to transfer the required data for the application. Hence, CAN can not be used for modern driver assistant applications.

\section{B. Local Interconnect Network (LIN) Bus}

LIN bus [4] is the youngest and general purpose serial system for low cost communication in cars. It was designed as an open standard and a cheap variant compared to CAN. LIN was introduced by a consortium of Motorola, Audi, BMW, Daimler, Volcano, VW and Volvo to offer low-cost communication for smart sensors and actors, which do not need the bandwidth and flexibility of a CAN bus.

LIN specification consists of the PHY, the communication protocol, application and interfaces for development tools. This comprehensive specification speeds up configuration and development of LIN networks. In the automotive domain LIN is used for example in door modules, sun roofs, controls at the steering wheel, seat control, heating and climate.

1) Advantages: LIN is a single wire communication protocol based on SCI (UART) 8 bit interface. UART interfaces are common available either in software or in firmware, on most microcontrollers. The system is a single master and multiple slaves variant. The bus master controls the arbitration, thus no collision management is needed.

2) Disadvantages: The maximum transfer speed is 20 kbit/s. Also the LIN bus is no standard bus system for camera manufacturers although in the automotive domain there are cameras connected by LIN bus with ECUs.

3) Recommendation: LIN has a similar recommendation as CAN. It is not possible to use LIN for camera based driver assistance systems. The bandwidth which can be used to send images from one camera to a special ECU is too small. 


\section{Time Triggered Protocol SAE Class C (TTP/C)}

TTP/C [5] [6] is a time-triggered communication protocol based on time division multiple access method (TDMA) and is used mainly in safety critical domains, e.g. avionics or Xby-Wire applications in the automotive domain. TTP uses predefined time slots which define the time when a node is allowed to send its message.

TTP/A is cheap variant of TTP/C as LIN is for CAN. Instead of TTP/C TTP/A does not support hard real-time requirements. TTP/A is a master-slave UART based protocol where the master provides the timing. Every slave sends its message only on a request initiated by the master and all communication between slaves is routed through the master.

1) Advantages: TTP/C is designed to fulfill the highest safety requirements. Its main advantage is composability: system components can be developed independent from each other. The maximum transfer speed is $10 \mathrm{Mbit} / \mathrm{s}$.

2) Disadvantages: The TTP/C protocol uses management nodes which manage the reconfiguration and reintegration of spare nodes if a failure has occurred. This increases the cost by using special purpose hardware and adds additional delay by integrating the spare nodes. Due to the time-triggered protocol, the maximum delay of a message is larger than that of CAN. Additionally the TTP/C system is not compatible with current event-triggered systems. Currently TTP/C nodes can only be bought from TTTech.

3) Recommendation: In camera based driver assistance systems bandwidth is one of the most important factor. TTP/C satisfies this requirement with a speed of $10 \mathrm{Mbit} / \mathrm{s}$. On the other side sending data only in an appropriate time slot and then waiting until all other systems have send their data is not a possibility. This means a loss of image data until the camera is allowed to send its new data which can be avoided by fine tuning of time slots and adding different nodes. Hence, this would imply a large engineering overhead. The biggest disadvantage is however the high cost for hardware parts and software tooling.

\section{FlexRay}

The time-triggered communication bus FlexRay [7] was developed by a combination of ByteFlight [8] and TTP/C (see III-C). As ByteFlight is only capable for passive safety systems, e.g. airbags, it is improper for active control systems by a lack of fault tolerance support, e.g. X-by-Wire. To overcome this problem BMW and Daimler founded the FlexRay consortium to improve ByteFlight.

The communication system allows a synchronous and asynchronous data transfer. The synchronous part is based on the TDMA method. Here, free configurable time slots with equal length are used. Every node is allowed to send a message which can be as long as the time slot or less. The asynchronous part is based on the ByteFlight protocol and uses the flexible time division multiple access method
(FTDMA). Every node can use the full bandwidth for eventtriggered data transfer.

1) Advantages: FlexRay guarantees minimal latency and jitter. Using a distributed clock synchronization redundancy, safety and fault tolerance is also supported. In the timetriggered part all network entities have got the same priority. This allows a deterministic calculation and prediction when a message will be sent or received. By doing so every entity knows its usable time intervals and hence collision are avoided. The minimal transfer speed is $10 \mathrm{Mbit} / \mathrm{s}$.

2) Disadvantages: Compared to TTP/C FlexRay is not composable. Thus during development and testing of one node or application all other parts of the network have to be either connected or to be simulated by special hardware. Additionally a complex tooling is needed to organize the time slots and the whole network system. FlexRay developers also need a comprehensive knowledge of all parameters to optimize such a system

3) Recommendation: FlexRay is designed to be used for fault tolerant environments as drive-by-wire or powertrain domains. Camera based systems will benefit from fault tolerant properties and all guarantees given by FlexRay. But the tooling and the knowledge of all parameters are main disadvantages of FlexRay. Developers of camera based systems want to optimize and improve their algorithms without dealing with specialties of a network.

\section{E. Low-Voltage Differential Signals (LVDS)}

LVDS [9]-[11] is an electrical digital signaling system that can run at very high speeds over inexpensive twistedpair copper cables. It was introduced in 1994 and has since become very popular in computers, where it forms part of very high-speed networks and computer buses. In the automotive industry LVDS is used for flat screens and camera data transfer. Therefore Open LVDS Digital Interface (OpenLDI) with a 36-pin Sub-D or MDR socket is used. OpenLDI supports high graphic resolutions of UXGA and higher with a color depth of 24 bits.

1) Advantages: LVDS is suited for high speed data transfer with a maximum transfer speed of $655 \mathrm{Mbit} / \mathrm{s}$ per channel. As given in [9] a maximum transfer speed of 1.923 $\mathrm{Gbit} / \mathrm{s}$ is theoretically achievable. LVDS also supports a maximum resolution of 2,048x 1,536 pixels.

2) Disadvantages: Depending on resolution and color depth the maximum data transfer length varies from 0.5 to 10 meters. There are only two big companies selling LVDS chips and a small group of manufacturers for cameras supporting LVDS. Additionally LVDS contains not an open protocol. The composition of components from different vendors is not supported and data exchange as to be done by ECUs acting as gateways.

3) Recommendation: According to resolution and maximum transfer speed LVDS is the state of the art for camera based driver assistance systems in the automotive industry. 
But due to the lack of an open protocol and a trend to reduce the number of network systems in a car, LVDS is one candidate which will be removed in near future.

\section{F. Media Oriented Systems Transport (MOST)}

MOST [12], [13] bus has been proposed as an automotive standard by the MOST Cooperation. The goal was to achieve a cheap Peer-to-Peer network system together with a suitable protocol for multimedia data without the use of a PC or other central ECUs. Where here multimedia is not only referring to audio and video data it also includes telecommunication, general purpose and control data. MOST can be used as either single-master or multi-master system.

1) Advantages: MOST supports up to 64 network nodes with plug-and-play capability. In the first version the maximum transfer rate is $24.8 \mathrm{Mbit} / \mathrm{s}$. But in the latest version, MOST150, a maximum speed of $150 \mathrm{Mbit} / \mathrm{s}$. MOST150 supports an interconnection with IP systems which allows using IP based applications to run without changes upon a MOST150 system.

2) Disadvantages: The first version can only be used with compressed video streams which do not exceed a maximum transfer rate of $3.1 \mathrm{Mbyte} / \mathrm{s}$. There are currently only a few manufacturers of MOST components or customizer of MOST components. At least there are some manufacturers for cameras using MOST bus as an interconnection.

3) Recommendation: The MOST bus emerged as the de-facto standard for telematics platforms. Therefore all currently developed camera based driver assistance systems have to support MOST bus. Due to high cost based on the single supplier problem and the disadvantage of a low maximum transfer speed compared to LVDS, MOST is not the preferred network for camera based driver assistance systems.

\section{G. Ethernet}

Ethernet [14], [15] is a family of network technologies and was commercially introduced in 1980. Every message is sent as a package with a maximum transmission unit (MTU) of 1500 bytes as said in version 2. Such packages are sent in frames which contains beside the data source and destination addresses, also error-checking data. Beside the wireless version Ethernet is currently not used in mass produced cars, but the automotive industry has started some research projects to test Ethernet in automobiles.

1) Advantages: Ethernet has a great commercial success, is cheap and provides connectivity to Internet. A lot of software has been developed to use Ethernet as a distribution system. The maximum transfer rate depends on the used variant, but currently Ethernet provide a speed of $1 \mathrm{Gbit} / \mathrm{s}$. In the automotive domain there is currently a special Ethernet version under development by Broadcom, called BroadRReach [16], or Micrel [17]. Manufacturers produce their camera systems either with an IEEE 1394 or an Ethernet connector. Thus a lot of different camera systems can be used.

2) Disadvantages: In the automotive industry size and weight matters therefore commonly used cables are not suitable. With the trend to reduce the number of network systems in cars one goal is to remove LVDS. So replacing one bus system, e.g. LVDS, by Ethernet only for camera based systems will not be done, even when the new system will not offer the disadvantages of the old one.

3) Recommendation: Currently both Broadcom and Micrel are combining the advantages of Ethernet with CAN in order to use dual wire cables instead commonly used cables. This would reduce weight and size of the cable but increase the prize of hardware especially the PHYs. Additionally this adapted Ethernet version supports a MTU of 1500 bytes, whereas camera based systems need a MTU of 9000, known as Jumbo frames. Without the Jumbo frame support, the adapted Ethernet will not be suitable for mass production.

\section{H. Audio Video Bridging (AVB)}

AVB [18] is developed by the IEEE 802.1 Audio Video Bridging Task Group. The goal is to provide a standard which allows transferring audio and video data digital for professional and private applications. Therefore the Ethernet standard has been improved by additional features like precise synchronization of multiple streams, bandwidth reservation and traffic management. In the automotive domain, AVB is currently tested to be one possible solution for telematics platforms, a replacement for e.g. MOST, and a solution for critical control applications, a replacement for e.g. FlexRay. An automotive requirement analysis for AVB is given in [19].

1) Advantages: AVB supports streaming of audio and video data. These streams can be synchronized even though they travel on different paths with different sample rates [20]. Additionally non AVB suitable hardware is also handled and streaming is adapted to that kind of problems.

2) Disadvantages: Although a lot of alliances and manufacturers are presenting their AVB demo hardware or claiming their goodwill, there is no mass market and the available hardware is too expensive for the automotive industry.

3) Recommendation: In the current situation AVB is not usable for camera based driver assistance systems.

\section{Conclusion}

Modern camera based driver assistance systems rely on a high bandwidth. The required bandwidth depends on the resolution and frames per second. Therefore communication systems with small bandwidth like LIN and CAN are not useful. Other systems are not designed to interact with cameras and their behavior. Both TTP/C and FlexRay are such systems which are normally not used to interact with cameras and do not provide so much bandwidth for high resolutions. 
Table I

A COMPARISON OF CURRENT AUTOMOTIVE NETWORKS

\begin{tabular}{|c|c|c|c|c|c|c|}
\hline & Bandwidth & max. Payload (in byte) & Topology & Real-Time & Costs & applicability \\
\hline $\mathrm{CAN}$ & $1 \mathrm{Mbit} / \mathrm{s}$ & 8 & multi-master & no & low & - \\
\hline LIN & $20 \mathrm{kbit} / \mathrm{s}$ & 8 & single-master & no & low & - \\
\hline $\mathrm{TTP} / \mathrm{C}$ & $10 \mathrm{Mbit} / \mathrm{s}$ & 128 & single-master & yes & high & - \\
\hline FlexRay & $10 \mathrm{Mbit} / \mathrm{s}$ & 254 & single-master & yes & medium & - \\
\hline LVDS & $1.923 \mathrm{Gbit} / \mathrm{s}$ & - & multi-master & no & low & + \\
\hline MOST & up to $150 \mathrm{Mbit} / \mathrm{s}$ & 1014 (MOST25), 3072 (MOST150) & single-master or multi-master & no & medium & + \\
\hline Ethernet & $1 \mathrm{Gbit} / \mathrm{s}$ & 1500 (Jumbo-Frames: 9000) & - & no & low & + \\
\hline AVB & $100 \mathrm{Mbit} / \mathrm{s}$ & 1500 & - & no & high & + \\
\hline
\end{tabular}

LVDS, Ethernet and AVB provide a flexible system with high bandwidth according to their specification. LVDS is the de facto standard for camera based systems but does not support an open interface. Therefore Ethernet is the choice for our demonstrator because a lot of software and hardware already exists. Additionally the hardware is cheap and supports high resolutions by the usage of Jumbo frames. AVB has not been chosen as there is currently no camera manufacturer.

\section{SIDE-VIEW SYSTEM}

Our Side-View system is a prototypical implementation of a camera based driver assistance system. This system is used as a demonstrator platform for IP based systems in SEIS. The system contains of five cameras. All cameras are normal GigE commercially available Off-The-Shelf (COTS)cameras. Two cameras are used for front and rear view. For the cameras mounted on both sides, a special setup is used. Because of the small space given in the side mirrors the cameras have been separated into the sensor, containing the lens, and the microcontroller, containing the Ethernet connector. Both parts are connected by a thin connector band such that the space of a side mirror is used optimal. Normally all the listed parts are combined in one case as it is with the front and rear camera. The last camera is a board camera without a case. This version of a GigE camera is used because it is integrated into the inside roof lining. A lot of driver assistance applications use lenses with a small flare angle. This satisfies all needs for road sign recognition or lane detection as an example. In our case a small flare angle will not work. Thus we use a so called fisheye lens. The flare angle of a fisheye lens can vary from 127 degree to 360 degree, but our use case uses fisheye lenses with a flare angle of 180 degree. This allows us to get a panoramic view of the whole environment around a car. The interconnection of the camera systems with each other is done by Ethernet. An IP network is used to control every single camera. Physically the cameras are connected to a manageable switch and through that to a Car-PC which can be later replaced by an ECU. Our Car-PC is equipped with an Intel Core i5 (2.5 GHz), 3.17 GByte RAM, a 128 GByte SSD and an Intel HD Graphic card. It runs a Windows XP

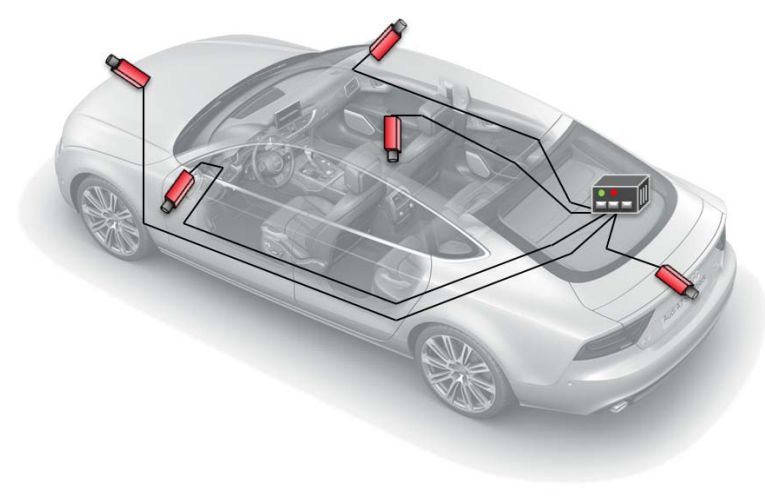

Figure 2. Side-View Demonstrator

and uses a VLC Media Player as a VLC Streaming Server. The Car-PC is used as an internal server to collect, buffer the data delivered by the camera systems and to do the image processing. Additionally we are using an iPad as a tablet PC which runs iOS and a version of VLC to get the camera streams. As a connector between the Car-PC and the tablet we set up a server system with an Apache, a VLC Streaming Server and Java with JMF. The server simulates a repair station system.

This setup allows the use of COTS technology in driver assistance applications instead of specific cameras for specific networks. Ethernet or, more precise, GigE cameras are widely used, so costs of such systems are decreasing. Additionally using Ethernet enables a simple and cheap wiring solution. With an Ethernet based system more software can be used without any adaption.

\section{SUMmary}

This paper provides a comprehensive overview of all state-of-the-art automotive communication systems which can be used for modern camera based driver assistance systems. The communication systems were presented and analyzed for their usefulness and appropriateness for modern camera based driver assistance systems. Recommendations 
were made for the best use of each of several common data communication buses.

Based on the choice of a communication bus which is suitable for modern camera based driver assistance systems the setup of the Side-View system was explained. In addition, two use cases were described which both use the Side-View system.

As next step we want to improve the system with a new image processing algorithm. First the single images will be combined to one panoramic view, in a similar way to the algorithm given in [21]. Additionally an image processing algorithm will scan each single image for person recognition and will measure the distance between the object and the vehicle. The detected object and its distance will be compared with a zone model to estimate the threat potential. SNMP traps will be installed to trigger an alarm if the threat is beyond a threshold. At the end we will do some measurements to optimize the system.

\section{ACKNOWLEDGMENT}

P. S. Correia and A. Camek want to thank Mr. Hanke and Mr. Seemann from AUDI AG for their support within this research project.

\section{REFERENCES}

[1] eNOVA Strategiekreis Elektromobilität, "SEIS - Sicherheit in Eingebetteten IP-basierten Systemen," 2010. [Online]. Available: http://www.strategiekreis-elektromobilitaet. de/public/projekte/seis

[2] R. V. White and D. Freeman, "Data Communications Issues For Power System Management," in Applied Power Electronics Conference, APEC 2007 - Twenty Second Annual IEEE, 25 2007-march 1 2007, pp. 1188 -1199.

[3] Robert Bosch GmbH, CAN Specification, September 1991.

[4] LIN Consortium, LIN Specification Package, November 2006.

[5] TTA-Group, Time-Triggered Protocol TTP/C High-Level Specification Document Protocol, version 1.1 ed., November 2003.

[6] H. Kopetz, M. Holzmann, and W. Elmenreich, "A universal smart transducer interface: TTP/A," in Object-Oriented RealTime Distributed Computing, 2000. (ISORC 2000) Proceedings. Third IEEE International Symposium on, 2000, pp. 16 -23 .

[7] FlexRay Consortium GbR, FlexRay Specification, May 2008.

[8] J. Berwanger, M. Peller, and R. Griessbach, A New HighPerformance Data Bus System for Safety-Related Applications, BMW AG, Knorrstrasse 147, 80788 Munich, Germany.

[9] IEEE Project 1596.3 Working Group, "IEEE Standard for Low-Voltage Differential Signals (LVDS) for Scalable Coherent Interface (SCI)," IEEE Std 1596.3-1996, p. i, 1996.

[10] National Semiconductor, LVDS Owner's Manual, 2008.
[11] Texas Instruments, LVDS Application and Data Handbook, 2002.

[12] MOST Cooperation, MOST Specification, May 2008.

[13] Prof. Dr. Ing. Andreas Grzemba, MOST - The Automotive Multimedia Network. Franzis Verlag GmbH, 2008.

[14] IEEE 802.3 Ethernet Working Group, "IEEE Standard for Information Technology -Telecommunications and Information Exchange Between Systems - Local and Metropolitan Area Networks - Specific Requirements Part 3: Carrier Sense Multiple Access With Collision Detection (CSMA/CD) Access Method and Physical Layer Specifications - Section One," IEEE Std 802.3-2008 (Revision of IEEE Std 802.3-2005), pp. c1-597, 262008.

[15] — - "IEEE Standard for Information technology - Telecommunications and information exchange between systemsLocal and metropolitan area networks - Specific requirements Part 3: Carrier Sense Multiple Access with Collision Detection (CSMA/CD) Access Method and Physical Layer Specifications - Section Five," IEEE Std 802.3-2005 (Revision of IEEE Std 802.3-2002 including all approved amendments), vol. Section5, pp. 1-417, 2005.

[16] Broadcom Corporation, BCM89810 - BroadR-Reach SinglePort Automotive Ethernet Transceiver, October 2008.

[17] Micrel Inc., Industrys First and Only AECQ-100 Automotive Qualified Ethernet Devices, October 2008. [Online]. Available: http://www.micrel.com/applications/auto/Ethernet_ Automotive.pdf

[18] Audio/Video Bridging Task Group, "Audio Video Bridging Systems," IEEE Std 802.1BA, vol. Draft 2.4, pp. 1 -, 2010.

[19] J. Takeuchi, H. Goto, S. Iiyama, T. Nomura, H. Kosugi, M. J. Teener, and Y. Kim, "Requirements for Automotive AVB System Profiles," Whitepaper, Knorrstrasse 147, 80788 Munich, Germany, March 2011. [Online]. Available: http://www.avnu.org/files/static_ page_files/9F0A4E3F-1D09-3519-ADBA4F0C747D7640/ ContributedAutomotiveWhitepaper_April2011.pdf

[20] R. Kreifeldti, A. Chang, A. J. Huotari, Y. Kim, K. Lewis, and K. B. Stanton, "AVB for Professional A/V Use," Whitepaper, Knorrstrasse 147, 80788 Munich, Germany, July 2009. [Online]. Available: http://www.meyersound.com/pdf/ products/d-mitri/AVnu_Pro_White_Paper_highres.pdf

[21] T. Gandhi and M. Trivedi, "Vehicle Surround Capture: Survey of Techniques and a Novel Omni-Video-Based Approach for Dynamic Panoramic Surround Maps," Intelligent Transportation Systems, IEEE Transactions on, vol. 7, no. 3, pp. 293 -308 , sept. 2006. 